\title{
BMJ Open Gender disparities in depressive and anxiety symptoms among internal migrant workers in Shenzhen: a cross- sectional study
}

\author{
Fengsu Hou (D) , ${ }^{1}$ Huiming Liu, ${ }^{1}$ Xiaodong Peng, ${ }^{1}$ Liqin You, ${ }^{1}$ Zhijian Zhou, ${ }^{1}$ \\ Haiyan Xie, ${ }^{1}$ Tiebang Liu $^{2}$
}

To cite: Hou F, Liu H, Peng X, et al. Gender disparities in depressive and anxiety symptoms among internal migrant workers in Shenzhen: a cross-sectional study. BMJ Open 2020;10:e041446. doi:10.1136/ bmjopen-2020-041446

- Prepublication history and additional materials for this paper is available online. To view these files, please visit the journal online (http://dx.doi. org/10.1136/bmjopen-2020041446).

FH and HL are joint first authors.

Received 09 June 2020

Revised 23 October 2020

Accepted 30 October 2020
Check for updates

(C) Author(s) (or their employer(s)) 2020. Re-use permitted under CC BY-NC. No commercial re-use. See rights and permissions. Published by BMJ.

${ }^{1}$ Department of Public Health, Shenzhen Kangning Hospital/ Shenzhen Mental Health Center, Shenzhen, Guangdong, China

2Shenzhen Kangning Hospital/ Shenzhen Mental Healch Center Shenzhen, Guangdong, China

Correspondence to

Professor Tiebang Liu;

liutbsz@126.com

\section{ABSTRACT}

Objectives To investigate the gender disparities in the prevalence and severity of depressive and anxiety symptoms and associated factors among internal migrant workers in Shenzhen.

Design Cross-sectional study.

Setting Labour intensive factories in Shenzhen, Guangdong, China.

Participants We recruited 3200 internal migrant workers who aged over 18 years old and above and did not register in Shenzhen's household registration system. There were 3095 participants eligible for this study.

Methods Participants completed sociodemographic questionnaire, the Patient Health Questionnaire-9, the Generalized Anxiety Disorder-7, the UCLA Loneliness Scale, the Barratt Impulsiveness Scale, the Social Support Rating Scale, the Simplified Coping Style Questionnaire and Meaning in Life Questionnaire. We applied $\chi^{2}$ test, analysis of variance, Wilcoxon rank test, Fisher's exact test and univariate and multivariate multilevel linear regression analysis.

Results The overall prevalence of depressive and anxiety symptoms was $27.85 \%$ and $19.26 \%$ among internal migrant workers. We reported gender disparities of depressive and anxiety symptoms among participants that the prevalence of depressive and anxiety symptoms was higher in women ( $30.57 \%$ vs $26.43 \%$ and $22.67 \%$ vs $17.47 \%$ ), and the symptoms were more severe among women. Female migrant workers were more likely to be singled, have lower prevalence of smoking and drinking, receive less education and monthly income, have higher level of impulsiveness and social support and lower level of meaning in life. We found age, marriage, income, adaption to living in Shenzhen, being discriminated, drinking, loneliness, impulsiveness, social support, coping strategies and meaning of life were associated with the severity of depressive and anxiety symptoms among internal migrant workers in Shenzhen. Conclusion Gender inequality may be the institutional factor leading to disparities in depressive and anxiety symptoms among internal migrant workers. Interventions should be embedded with strategies improving gender equality.

\section{INTRODUCTION}

Internal migrant workers have made important contributions to China's
Strengths and limitations of this study

- This is a cross-sectional study with a large sample of Chinese internal migrant workers in Shenzhen exploring the gender disparities in the prevalence and severity of depressive and anxiety symptoms.

- We report factors associated with the severity of depressive and anxiety symptoms among Chinese internal migrant workers.

- This study recruited participants from labour intensive factories that limited the generalisability to internal migrant workers in other industries.

- We were limited to detailed information on gender disparities and could not conceptualise frameworks to explain the mechanism from gender disparities to mental health problems.

accomplishments in economics, industrialisation and urbanisation in recent decades. In 2018, the internal migrant population has been up to 244 million, accounting for $17.4 \%$ of China's total population. ${ }^{1}$ Based on China's household registration system, the 'Hukou' policy, migrant workers or the 'floating population' are defined as people who leave their registered residence areas (eg, cities, towns and villages) for engaging in various jobs in non-residence areas. The coastal urban cities, like Shenzhen in the Pearl River Delta area, are the major destinations of the internal migration.

Migrant workers are vulnerable to both physical and psychological problems because of the 'Hukou' policy that migrants do not share the equal social benefits as the registered household residents, including education, employment, healthcare and social services in urban cities. ${ }^{2}{ }^{3}$ After a series of Foxconn migrant worker suicides in Shenzhen, 2010, the mental health of migrant workers in China, especially depression, anxiety and suicide, has gained tremendous 
attention; and a growing number of studies have examined the relationship between internal migration and mental health through different perspectives, such as help-seeking, income-related inequality and social integration. ${ }^{4-9}$ The prevalence of mental health problems of migrant workers varies among inland and coastal urban cities. For example, the prevalence of depressive symptoms varied from $16.5 \%$ in Beijing, $23.7 \%$ in Chengdu, $34.2 \%$ in Wuxi to $37.3 \%$ in Shenzhen. ${ }^{10-13}$

Women are in greater risk for mental health problems than men. ${ }^{14} 1^{5}$ The increased risk cannot be simply attributed to biological differences, it results from the interactions between biological factors and social determinants including gender stereotypes and roles, social stigma and inequality, and social autonomy. ${ }^{16}{ }^{17}$ Internal migration and related changes naturally serve as their primary source of stressors for accumulative stress leading to mental health problems. ${ }^{18}$ In He and Wong' s study of 959 female migrant workers from 12 factories in Shanghai, Kunshan, Dongguan and Shenzhen, about $24 \%$ of participants were in poor mental health, measured by the Brief Symptom Inventory, and the rate was the highest in Shenzhen (35\%) ${ }^{19}$ Other studies, applying the Symptom Check List 90 (SCL-90), reported female migrant workers gained higher scores in most of the subscales than the Chinese norms, and the prevalence of any mental health symptoms was also higher than the men. ${ }^{20}{ }^{21}$ Few studies reported the prevalence of a specific mental health problem among female migrant workers. For example, in Beijing, a study reported the prevalence of depression was $22.6 \%$ among female migrant workers, which was close to another study in Shenzhen reporting the prevalence was $22.4 \%^{22}{ }^{23}$; and another study in Chengdu reported the prevalence of anxiety was $22.72 \%$ among female migrant workers, and there was no gender differences. ${ }^{24}$

Shenzhen is one of the pilot cities in China to develop the Psychosocial Service System (PSS). There were about 8.48 million internal migrants in Shenzhen accounting for $65.1 \%$ of its total population in 2018, and a large portion of the migrants working in labour-intensive industries have gained great attention in the PSS development. ${ }^{25}$ This study is a part of the Social Epidemiological and Biological Study of Suicide Behaviors Among Factory Migrant Workers in Shenzhen, which aims to investigate the social, biological and mental health factors associated with suicide, suicide ideation, suicide plan and suicide attempts among internal migrant workers in Shenzhen. The current study aims (1) to investigate the gender disparities in the prevalence and severity of depressive and anxiety symptoms among internal migrant workers in Shenzhen; (2) to explore factors associated with the severity of depressive and anxiety symptoms.

\section{METHODS}

\section{Sample and sampling}

This study adopted the sample of the parent study, and the sample size was calculated to estimate the prevalence of suicide ideation among internal migrant workers in Shenzhen based on the following equation:

$$
n=Z_{1-a / 2}^{2} p(1-p) / d^{2}
$$

Based on a prior study that reported the prevalence of suicide ideation was $19.9 \%$ among internal migrant workers in Shenzhen, ${ }^{26}$ the parent study set the significant level $\alpha$ at 0.01 , the quantity $d$ for permissible error as 0.02 and the sample size was 2644; meanwhile, considering the possible clustering effect and sample loss, the parent study used a positive design effect of 1.20 to set the adjusted sample size as 3200 .

During 2018-2019, with a multistage sampling strategy, the parent study first randomly selected 4 out of 10 districts in Shenzhen, then randomly selected 8 labour intensive factories to recruit participants. For each factory, the parent study randomly selected 400 participants. The parent study recruited migrant workers who: (1) aged 18 years old and above, (2) were born elsewhere or did not register in Shenzhen's Hukou (household registration) system, (3) provided written consent. Internal migrant workers who had a history of severe mental disorders that might impede completing the survey were excluded.

\section{Procedure}

Before the field survey, the study team contacted with liaisons in selected factories, and the liaisons delivered written consents to selected migrant workers based on the sampling frame. The study team and the liaisons determined a date and gathered participants to finish the field survey after participants provided consents. Considering mental health problems were sensitive information, we required all participants to complete the survey while keeping social distance.

During the field survey, experienced and well-trained investigators helped participants complete questionnaires and reminded participants to complete missing items.

\section{Measurements}

The study team developed the sociodemographic questionnaire to collect participants' characteristics including age, gender, education, marriage, monthly personal income, length of staying in Shenzhen, adaption, selfperceived discrimination, drinking, smoking and number of mental health source.

We applied the Chinese version of Patient Health Questionnaire-9 (PHQ-9) to measure the severity of depressive symptoms, which has shown great reliability and validity. ${ }^{27} 28$ The items capture nine symptom criteria for clinical depression diagnosis from Diagnostic and Statistical Manual of Mental Disorders, Fourth Edition (DSMIV). Each item can be scored from 0 ('not at all') to 3 ('nearly every day'), and the total score ranges from 0 to 27 with a higher score indicating a more severity of depressive symptoms; and a total score of 5, 10, 15 and 20 indicates mild, moderate, moderately severe and severe 
depression. ${ }^{27}$ We set the cut-off point at 5 to screen for depressive symptoms. The Cronbach's alpha was 0.880 in this study.

We applied the Chinese version of the Generalized Anxiety Disorder-7 (GAD-7) measure the severity of anxiety symptoms, which has shown great reliability and validity. ${ }^{29}$ The times reflect all symptom criteria for GAD from DSM-IV. Each item can be scored from 0 ('not at all') to 3 ('nearly every day'), and the total score ranges from 0 to 21 with a higher score indicating a more severity of anxiety symptoms; and a total score of 5, 10 and 15 indicates mild, moderate and severe anxiety. ${ }^{29}$ We set the cut-off point at 5 to screen for anxiety symptoms. The Cronbach's alpha was 0.906 in this study.

We applied the Chinese version of 6-item UCLA Loneliness Scale (ULS-6) to measure loneliness, which has been translated and validated in China. ${ }^{31}{ }^{32}$ The total score ranges from 6 to 24 with a higher score indicating a more severity of loneliness. The Cronbach's alpha was 0.859 in this study.

We applied the Chinese version of the Barratt Impulsiveness Scale (BIS-11) to measure impulsiveness, which has been translated and validated in Chinese population. ${ }^{33}$ The total score ranges from 0 to 100 with a higher score indicating a higher level of impulsiveness. The Cronbach's alpha was 0.794 in this study.

We applied the Social Support Rating Scale (SSRS) to measure social support, which was a 10-item scale developed and has been widely used in China with great reliability and validity. ${ }^{34-37}$ The total ranges from 12 to 66 with a higher score indicating a higher level of social support. The Cronbach's alpha was 0.804 in this study.

We applied the Simplified Coping Style Questionnaire to explore how participants cope with daily stress or events, which was 20-item scale developed in China. ${ }^{38}$ There are two subscales, positive coping and negative coping, and a higher mean score of each subscale indicates being more inclined to adopt the coping strategy. The Cronbach's alpha was 0.863 in this study.

We applied the Chinese version of Meaning in Life Questionnaire (C-MLQ) to measure how participants assessed the presence of and searched for meaning in life, which was a 10-item scale and had been translated and validated in China. ${ }^{39} 40$ The total score ranges from 10 to 70 with a higher score indicating more satisfied in life. The Cronbach's alpha was 0.844 in this study.

\section{Statistical analysis}

In this study, we analysed data with R (V.3.5.1) and set the statistical significance at $0.05{ }^{41}$

\section{Data preparation}

Before analysis, we recoded participants' marital status into two categories: singled and married/coupled. We categorised participants' education level into four groups: primary school and below ( $\leq 6$ years of education), junior high school (7-9 years of education), high school (10-12 years of education) and college and above ( $\geq 13$ years of education). We categorised participants' personal monthly income into four groups: $\leq \$ 439.49$ (¥2999), \$439.64-\$732.58 (¥3000-¥4999), \$732.73-\$1465.31 (¥5000-¥9999) and $\geq \$ 1465.46$ (¥10 000)。

\section{Analytic plan}

To compare the characteristics between male and female participants, we applied one-way analysis of variance or Wilcoxon rank test (if the data were of skewed distribution) for continuous variables, and $\chi^{2}$ test for categorical variables. Descriptive analysis was conducted by $\mathrm{R}$ package 'psych'. ${ }^{42}$

We created dummy variables for categorical variables, and the first category of each variable the reference group was the reference group in regression analysis. Considering the clustering effect of the sample, we conducted univariate multilevel linear regression analysis between the severity of depressive symptoms/anxiety symptoms and potential associated variables including sociodemographic factors, loneliness, impulsiveness, social support, coping strategy and meaning of life, and then to conduct multivariate multilevel linear regression analysis. In multivariate analysis, we adopted a stepwise backward strategy, and the baseline model was the first model with explanatory variables that showed significance in the univariate analysis $(p<0.05)$. We chose the Akaike information criterion (AIC), the Bayesian information criterion (BIC) and adjusted R-squared and to assess the fitness of models, and lower values of the parameters indicated better model fitness. Analysis were conducted by R package 'car' and 'Ime4' and 'MASS'. ${ }^{43-45}$

\section{Patient and public involvement}

This study was conducted without patient and public involvement.

\section{RESULTS}

The parent study recruited 3200 participants, and there were 105 participants did not provide complete information on PHQ-9 or GAD-7, who were excluded from analysis in this study. Of 3095 eligible participants, there were 2032 men and 1063 women. The differences of sociodemographic information between eligible and non-eligible participants were not significant.

Overall, the age of participants ranged from 18 to 62 years old with a mean of $34.38 \pm 9.03$. There were $1959(63.30 \%$, $1959 / 3095)$ participants being married or coupled. There were $47.21 \%(1461 / 3095)$ and $43.49 \%(1346 / 3095)$ of participants have finished junior high school and high school. A majority of participants $(64.46 \%, 1995 / 3095)$ received monthly personal income between $\$ 439.64$ and $\$ 732.58$. The length of stay in Shenzhen ranged from 1 month to 34 years with a mean of $7.53 \pm 6.17$ years. There were $89.63 \%$ of participants $(2774 / 3095)$ adapted to living in Shenzhen, and there were $75.12 \%$ of participants $(2325 / 3095)$ reported not being discriminated. The prevalence of smoking and drinking was $31.21 \%$ (966/3095) 
and $42.58 \%$ (1318/3095), respectively. The total number of mental health resource ranged from 0 to 9 with a mean of $1.35 \pm 0.95$. The score of ULS- 6 ranged from 6 to 24 with a mean of $9.89 \pm 3.86$. The score of BIS-11 ranged from 25 to 88 with a mean of $51.43 \pm 8.78$. The score of SSRS ranged from 14 to 63 with a mean of $38.85 \pm 8.63$. The score of positive coping ranged from 0 to 36 with a mean of 21.39 \pm 7.47. The score of negative coping ranged from 0 to 24 with a mean of $8.14 \pm 4.72$. The score of C-MLQ ranged from 10 to 70 with a mean of $49.94 \pm 10.95$. The score of PHQ-9 ranged from 0 to 27 with a mean of $3.31 \pm 4.34$; and the prevalence of depressive symptoms was $27.85 \%$ (862/3095). The score of GAD-7 ranged from 0 to 21 with a mean of $2.30 \pm 3.53$; and the prevalence of anxiety symptoms was $19.26 \%(596 / 3095)$.

We observed gender disparities in several aspects. First, comparing with female participants, we found male participants were more likely to be singled $(43.90 \%$ vs $22.95 \%$ ), to receive high school education and above $(55.56 \%$ vs $42.17 \%)$, to receive personal income over $\$ 732.73$ ( $¥ 5000)$ ( $21.8 \%$ vs $11.1 \%$ ), to smoke $(46.31 \%$ vs $2.35 \%)$ and to drink (53.48\% vs $21.07 \%)$. We also found male participants were less impulsive, reported lower social support, and were more satisfied in life. Further, we reported the mean score of PHQ-9 (3.66 vs 3.14) and GAD-7 (2.69 vs 2.09) were higher in women than in men, and the prevalence of depressive and anxiety symptoms were also higher among women $(30.57 \%$ vs $26.43 \%$ and $22.67 \%$ vs $17.47 \%$ ). More details were shown in table 1 .

\section{Linear regression analysis of depressive symptoms}

Table 2 showed the results of univariate multilevel linear regression analysis for depressive symptoms. We found age, marriage status, monthly personal income, years in Shenzhen, adaption, discrimination, smoking, drinking, number of mental health source, loneliness, impulsiveness, social support, coping strategies and meaning of life were associated with depressive symptoms. Therefore, we included these variables into multivariate multilevel linear regression analysis (model 1 ).

In model 1, the AIC was 16 337.21, the BIC was 16 457.10 , and the adjusted R-squared was 0.4020 . We stepwisely removed years in Shenzhen, smoking, number of mental health source and positive coping strategy from the analysis. Finally, we got the final model (model 2). Comparing with model 1, model 2 improved in model fitness with an AIC of 16 331.71, a BIC of 16428.31 and an adjusted R-squared of 0.4003 .

Table 3 showed that the severity of depressive symptoms would increase $0.46,0.086$ and 0.11 unit for each unit of loneliness, impulsiveness and negative coping increased, respectively. Comparing with singled participants, the severity of depressive symptoms among married/coupled participants would be 0.26 unit higher; comparing with participants with monthly personal income of $\$ 439.49$ and below, the severity of depressive symptoms among those with income over $\$ 1465.46$ would be 2.30 units higher; and the severity of depressive symptoms would be 0.87 unit higher among participants did not adapt to living in Shenzhen. The severity of depressive symptoms would decrease 0.045, 0.022 and 0.015 unit for each unit of age, social support and meaning of life increased. Comparing with participants who reported discrimination and who reported drinking, the severity of depressive symptoms among those who did not report discrimination and did not drink would be 0.33 and 0.26 unit lower.

\section{Linear regression analysis of anxiety symptoms}

Table 2 showed the results of univariate multilevel linear regression analysis for anxiety symptoms. We found age, marriage, monthly personal income, years in Shenzhen, adaption, discrimination, drinking, number of mental health source, loneliness, impulsiveness, social support, coping strategies and meaning of life were associated with anxiety symptoms, and we included these variables into multivariate multilevel linear regression analysis (model 3).

In model 3, the AIC was 15 121.74, the BIC was 15 236.45, and the adjusted R-squared was 0.3845 . We stepwisely removed years in Shenzhen, drinking, number of mental health source, social support, positive coping strategy and meaning of life from the analysis. Finally, we got the final model (model 4). Comparing with model 3 , model 4 improved in model fitness with an AIC of 15116.08 and a BIC of 15194.57 .

Table 4 showed that the severity of anxiety symptoms would increase $0.42,0.065$ and 0.080 unit for each unit of loneliness, impulsiveness and negative coping increased, respectively. Comparing with participants with monthly personal income of $\$ 439.49$ and below, the severity of anxiety symptoms among those with income over $\$ 1465.46$ would be 1.57 units higher; and the severity of anxiety symptoms would be 0.38 unit higher among migrant workers did not adapt to living in Shenzhen. Comparing with participants who reported discrimination, the severity of anxiety symptoms among those who did not report discrimination would be 0.23 unit lower.

\section{DISCUSSION}

We identified several key findings based on a sample of 3095 internal migrant workers in Shenzhen, China: (1) the overall prevalence of depressive and anxiety symptoms was $27.85 \%$ and $19.26 \%$, which was lower than the previous study in Shenzhen ${ }^{13}$; (2) gender disparities were observed that the prevalence of depressive and anxiety was higher in women, and the symptoms were also more severe in them; (3) age, marriage, income, adaption to living in Shenzhen, being discriminated, drinking, loneliness, impulsiveness, social support, coping strategies and meaning of life were associated with the severity of depressive or anxiety symptoms; (4) we observed gender disparities among sociodemographic characteristics and psychological factors that male migrant workers were older, more likely to be married, to receive more education and income, to feel being discriminated, to drink, 
Table 1 Demographic information of participants

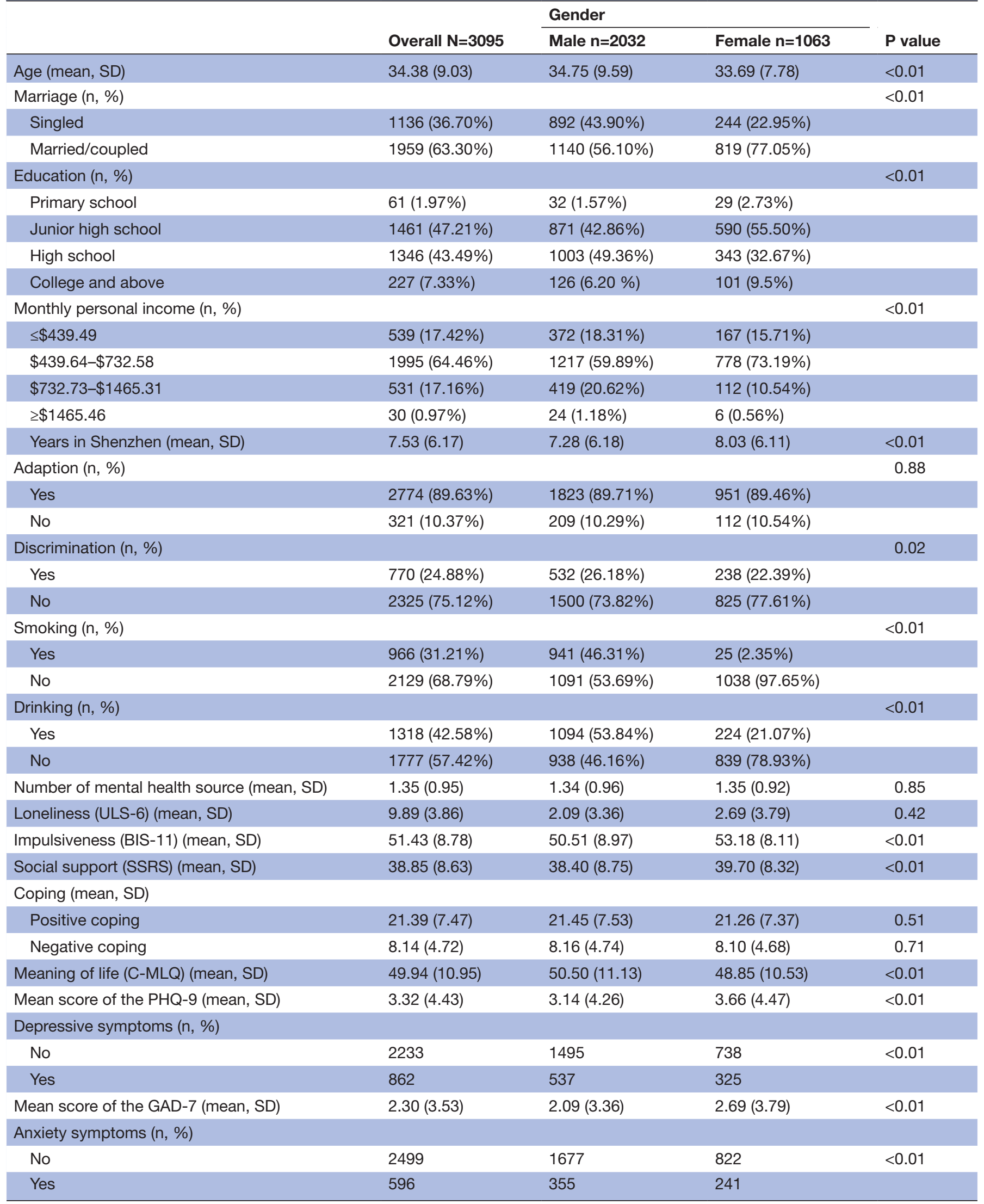

BIS-11, Barratt Impulsiveness Scale; C-MLQ, Chinese version of Meaning in Life Questionnaire; GAD-7, Generalized Anxiety Disorder-7; PHQ-9, Patient Health Questionnaire-9; SSRS, Social Support Rating Scale; ULS-6, 6-item UCLA Loneliness Scale. 
Table 2 Results of univariate multilevel linear regression analysis for depressive and anxiety symptoms

PHQ-9 score

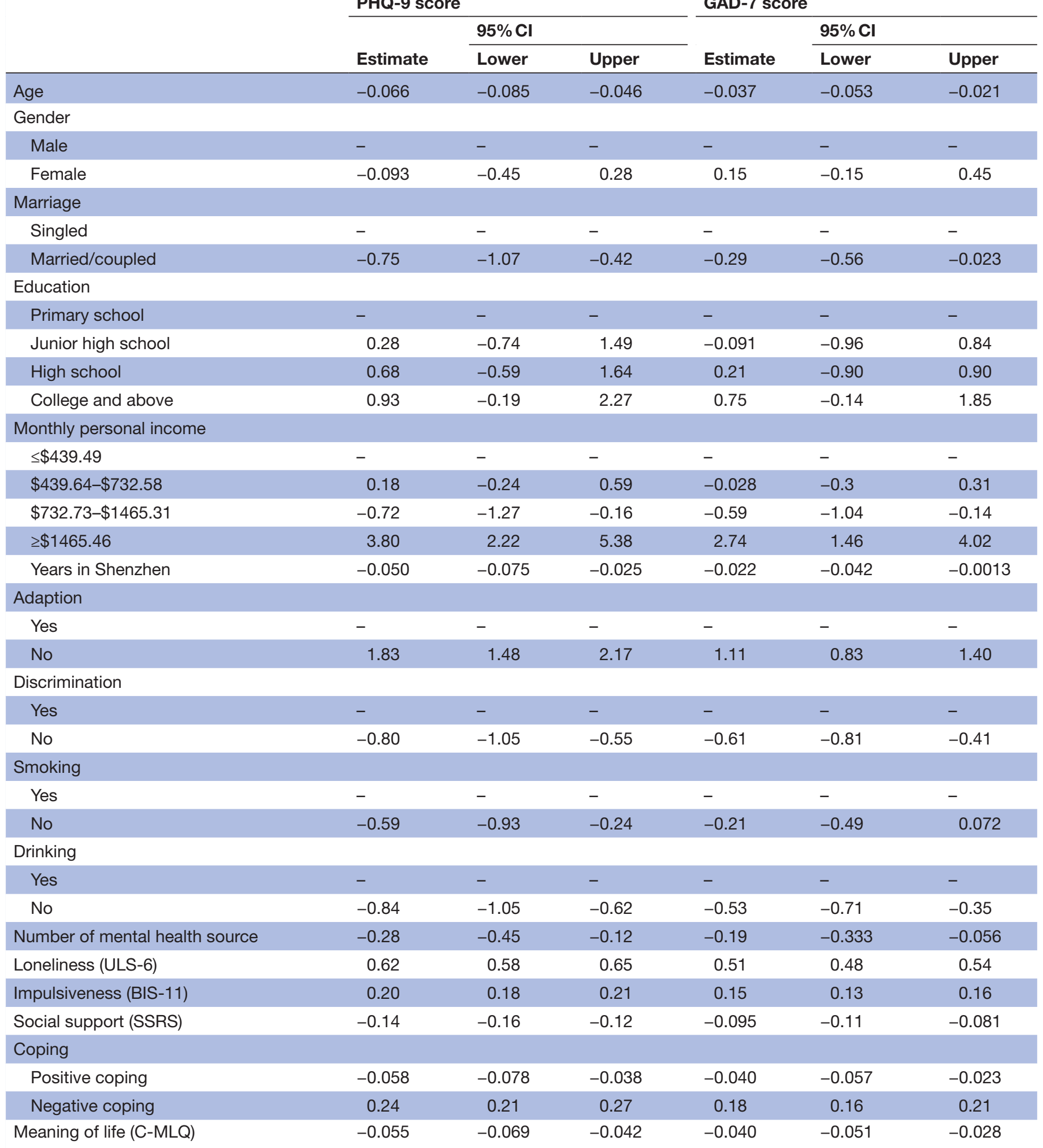

BIS-11, Barratt Impulsiveness Scale; C-MLQ, Chinese version of Meaning in Life Questionnaire; GAD-7, Generalized Anxiety Disorder-7; SSRS, Social Support Rating Scale; ULS-6, 6-item UCLA Loneliness Scale.

to be more satisfied with life, and to have a lower level of impulsiveness and social support.

To understand the mental health problems among internal migrant workers in China, it is necessary to be familiar with the 'Hukou' system. The 'Hukou' system, known as the household registration system, is implemented to classify the place of registration (urban or rural residence areas) and the type of registration (agriculture 
Table 3 Results of the stepwise multilevel linear regression analysis of depressive symptoms

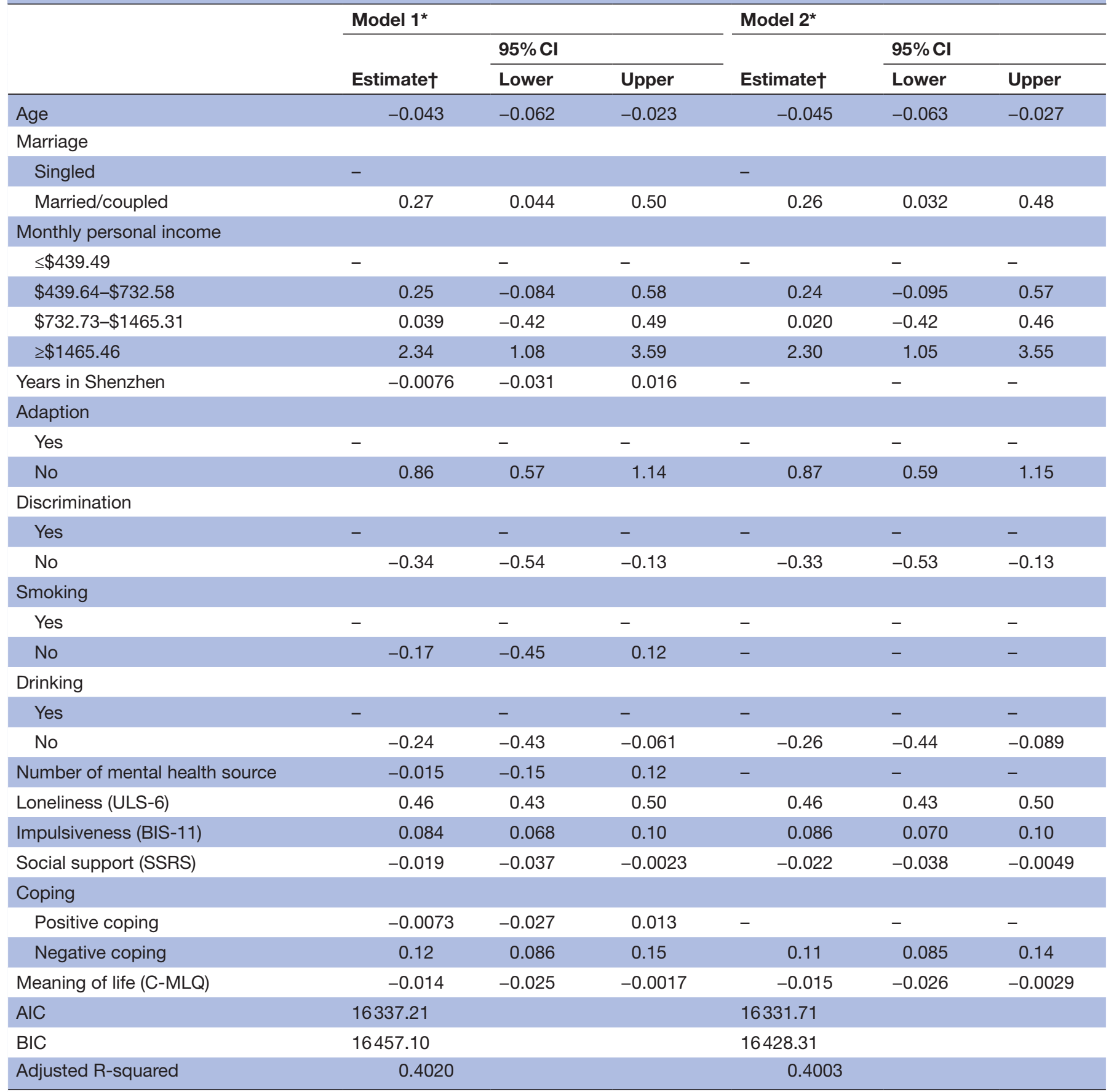

*Model 1 is the initial model of the multilevel linear regression analysis. Model 2 is the final model of the analysis after four iterations. †Estimate stands for the coefficient of each variable.

AIC, Akaike information criterion; BIC, Bayesian information criterion; BIS-11, Barratt Impulsiveness Scale; C-MLQ, Chinese version of Meaning in Life Questionnaire; SSRS, Social Support Rating Scale; ULS-6, 6-item UCLA Loneliness Scale.

or non-agriculture). ${ }^{46}$ Due to the registration, internal migrant workers, who are usually rural to urban migrants, have limited access to social welfare provided by the local governments of their destinations. Empirical studies have reported the associations between mental health and social inequities resulted from the registration system, including labour rights, wages, employment benefits, reimbursement for healthcare, limited access to public schools for migrant children. ${ }^{2-13}$

Noticeably, internal migrant workers in Shenzhen are faced with increased mental health problems than local community residents. A large epidemiology study among community residents from seven Chinese provinces reported the mean score of PHQ-9 and GAD-7 was 3.95 
Table 4 Results of the stepwise multilevel linear regression analysis of anxiety symptoms

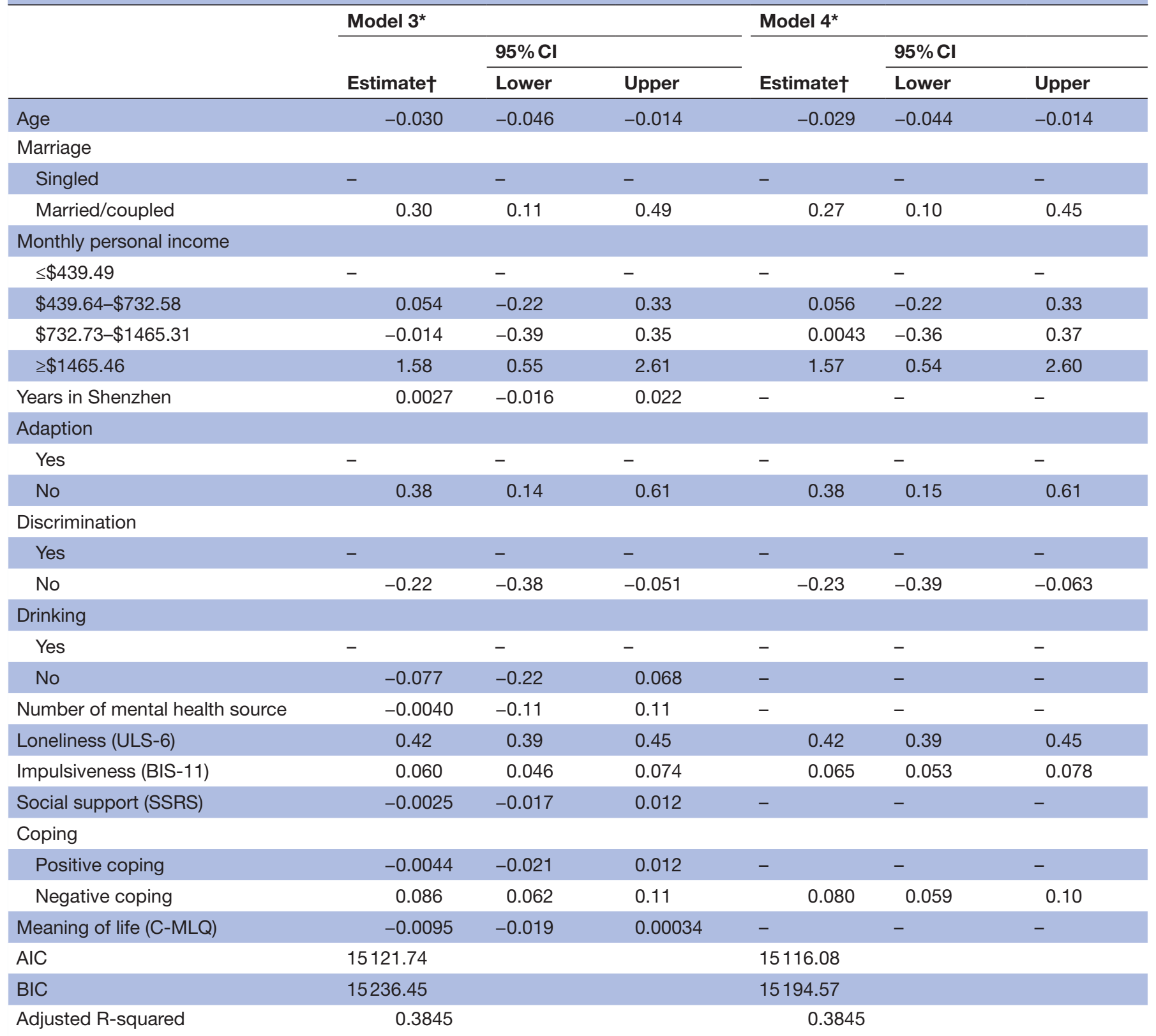

${ }^{*}$ Model 3 is the initial model of the multilevel linear regression analysis. Model 4 is the final model of the analysis after six iterations. †Estimate stands for the coefficient of each variable.

AIC, Akaike information criterion; BIC, Bayesian information criterion; BIS-11, Barratt Impulsiveness Scale; C-MLQ, Chinese version of Meaning in Life Questionnaire; SSRS, Social Support Rating Scale; ULS-6, 6-item UCLA Loneliness Scale.

and 2.71, respectively, and it also reported the score of GAD-7 was higher in women than in men $(2.75$ vs 2.66$) .{ }^{47}$ Further, this study comprised a sample of 2002 residents in Guangdong province and the mean score of PHQ-9 and GAD-7 in the subsample was 2.46 and 1.91 , respectively, and gender differences were not reported in the subsample. ${ }^{47}$ In comparison, we reported the mean score of PHQ-9 and GAD-7 among migrant workers in Shenzhen was 3.31 and 2.30, respectively, which were both higher than that in the mentioned study. An epidemiology study in 2009, applying the Composite International Diagnostic Interview, reported the prevalence of depression and anxiety in Shenzhen was $9.15 \%$ and $12.58 \%$ among registered residents, and it was $9.74 \%$ and $14.92 \%$ among non-registered residents. ${ }^{48}$ However, because of the nondiagnostic tools this study applied, we were limited to compare results.

The prevalence of depression and anxiety, including depressive and anxiety symptoms, varies among studies cross China, and we contribute the variation to following explanations. First, cross-sectional studies applied different tools to screen for depression and anxiety, such as the Center of Epidemiologic Studies Depression, the SCL-90, the Self-rating Depression Scale, the PHQ-9, the 
Beck's Depression Inventory, the GAD-7, the Self-rating Anxiety Scale and the Beck's Anxiety Inventory. ${ }^{10-13} 4749-51$ And even applying the same scale, studies may choose different cut-off points to report the prevalence, for example, we chose the cut-off point at 5 for PHQ-9 and GAD-7 while Wang set the cut-off point at 7 for both scales. ${ }^{47}$ Second, studies recruited different subgroups of Chinese internal migrant workers. In this study, participants came from labour intensive factories living in factory campuses which were micro-societal systems; other studies recruited participants from different industries like catering, retail and service and so on. Third, the prevalence also varies cross different samples of internal migrant workers because of sampling methods and sample size. Fourth, more developed cities, like first-tier cities (ie, Beijing, Shanghai, Guangzhou and Shenzhen), are selective based on migrants' skills, where working and living are much more stressful than the rest. ${ }^{52}$

We identified factors associated with depressive and anxiety symptoms from the social ecological framework, and our results were consistent with previous studies that lower sociodemographic status was associated with internal migrant workers' mental health problems. ${ }^{10} 5354$ Gender as a factor at the individual level, it crosses all levels of the framework and results in institutional effects leading to the gender disparities in mental health among internal migrant workers. Empirical studies among Chinese internal migrant workers reported that female migrant workers were younger, less educated and paid $20 \%-30 \%$ less than their male counterparts. ${ }^{755} 56$ Generally, rural households have lower educational expectations for girls, especially among poorer households, that lead to a higher dropout rate for girls ${ }^{57}$; and shortened education indicates women are younger and less skilled when they enter the labour market in urban cities resulting in the inequality of wages. ${ }^{58}$ We found female migrant workers have stayed longer in Shenzhen than the men, which may enable them a longer time to build social networks to increase their social support and reduce perceived discrimination in return. We reported female migrant workers perceived lower meaning in life, and it may result from the labour intensity and the inequality in wages. We believe the institutional gender disparities or even inequities might play an important role, and we encourage future research to collect detailed information, hypothesise the mechanism between mental health problems and gender disparities in sociodemographic factors, and test these hypotheses.

We recognise a few limitations of this study. First, the parent study did not aim to investigate the prevalence of depression and anxiety (including depressive and anxiety symptoms) among internal migrant workers from labour intensive factories in Shenzhen, and the sample did not recruit migrant workers from other industries; hence, it was difficult to estimate the representativeness of our findings comparing with the whole migrant worker population in Shenzhen. Second, because the parent study did not focus on gender disparities among migrant workers, we did not collect further information such as disparities in labour intensity, living environment, economic pressure, work related stress, and, especially, the interaction between gender disparities and the 'Hukou' system, hence we could not conceptualise frameworks to explain the mechanism from gender disparities to mental health problems. Third, as a cross-sectional study, we could not draw causal inferences from the findings. We encourage future studies to use longitudinal design to investigate the causal effects of gender disparities on migrant workers' mental health to develop strategies to improve migrant workers' mental health.

\section{CONCLUSION}

Among internal migrant workers in Shenzhen, women reported higher prevalence and severity of depressive and anxiety symptoms than the men, and the differences are associated with disparities resulting from institutional gender inequality. Interventions to improve the mental health of internal migration population in China should be embedded with strategies improving gender equality from individual to societal perspectives.

Acknowledgements We sincerely thank all participants who completed the survey. We sincerely acknowledge coordination work of liaisons from selected factories.

Contributors FH developed the plan for analysis, analysed the data, drafted and revised the paper. H-ML designed the survey instruments, monitored data collection, developed the plan for analysis and revised the paper. XP, LY, ZZ and HX designed the survey instruments, sent out recruitment advertisement, assisted in data collection and revised the paper. TL initiated the project, designed the study, and revised the paper. All authors had full access to all the data in the study and take responsibility for the integrity of the data and the accuracy of the data analysis. All authors read and approved the final manuscript.

Funding This study is funded by the Science and Technology Plan of Shenzhen Science and Technology Innovation Commission (JCY20170413101017457), and TL is the $\mathrm{PI}$ of the project.

Competing interests None declared.

Patient and public involvement Patients and/or the public were not involved in the design, or conduct, or reporting, or dissemination plans of this research.

Patient consent for publication Not required.

Ethics approval The Ethics Committee of Shenzhen Kangning Hospital reviewed and approved the protocol, including the online informed consent process, and approved analysis of de-identified data (KN-2020-04).

Provenance and peer review Not commissioned; externally peer reviewed.

Data availability statement Data are available upon reasonable request. The data on which this manuscript is based are not available to public. The data from this study are under certain restrictions according to the Shenzhen Science and Technology Innovation Commission and always under the supervision of the principal investigator of the study. Thus, there are access restrictions to the data. However, at any time, researchers can contact the principal investigator (Tiebang Liu, liutbsz@126.com) for data sharing.

Open access This is an open access article distributed in accordance with the Creative Commons Attribution Non Commercial (CC BY-NC 4.0) license, which permits others to distribute, remix, adapt, build upon this work non-commercially, and license their derivative works on different terms, provided the original work is properly cited, appropriate credit is given, any changes made indicated, and the use is non-commercial. See: http://creativecommons.org/licenses/by-nc/4.0/.

ORCID iD

Fengsu Hou http://orcid.org/0000-0001-5869-4365 


\section{REFERENCES}

1 National Health Commission of the People's Republic of China. China migrant population development report 2018. Beijing: National Health Commission of the People's Republic of China, 2018.

2 Keung Wong DF, Li CY, Song HX. Rural migrant workers in urban China: living a marginalised life. Int J Soc Welf 2007;16:32-40.

3 Chan KW. Migration and development in China: trends, geography and current issues. Migr Dev 2012;1:187-205.

4 Phillips MR. Foxconn and China's suicide puzzle. Wall Street J 2010;13.

5 Lam KKF, Johnston JM. Depression and health-seeking behaviour among migrant workers in Shenzhen. Int J Soc Psychiatry 2015;61:350-7.

6 Lin Y, Zhang Q, Chen W, et al. Association between social integration and health among internal migrants in Zhongshan, China. PLoS One 2016;11:e0148397.

7 Shao C, Meng X, Cui S, et al. Income-related health inequality of migrant workers in China and its decomposition: an analysis based on the 2012 China labor-force dynamics survey data. J Chin Med Assoc 2016;79:531-7.

8 Zhong B-L, Chan SSM, Liu T-B, et al. Mental health of the old- and new-generation migrant workers in China: who are at greater risk for psychological distress? Oncotarget 2017;8:59791-9.

9 Wen M, Zheng Z, Niu J. Psychological distress of rural-to-urban migrants in two Chinese cities: Shenzhen and Shanghai. Asian Popul Stud 2017;13:5-24.

10 Qiu P, Yang Y, Chen Q, et al. Depression and its impact factors among migrant workers in Chengdu. Modern Preven Med 2010;37:4263-6.

11 Chen Z, Zhang X, Chen X, et al. Relationship between depression and self-rated health among floating populations. Chinese $J$ Health Educ 2006;22:747-9.

$12 \mathrm{Xu}$ Y, Ji Y, Yuan Y, et al. Depressive symtoms and related factors among floating population. Chinese Mental Health J 2012;26:112-7.

13 Ding J, Zhou Z, Liu T, et al. Depression, anxiety and suicide risk among migrant workers in Shenzhen. 2012 annual Guangdong preventive Medicince academic conference compilation. Guangzhou: Guangdong Preventive Medicine Association, 2013.

14 GBD 2017 Disease and Injury Incidence and Prevalence Collaborators. Global, regional, and national incidence, prevalence, and years lived with disability for 354 diseases and injuries for 195 countries and territories, 1990-2017: a systematic analysis for the global burden of disease study 2017. Lancet 2018;392:1789-858.

15 Riecher-Rössler A. Prospects for the classification of mental disorders in women. Eur Psychiatry 2010;25:189-96.

16 Afifi M. Gender differences in mental health. Singapore Med J 2007;48:385.

17 Riecher-Rössler A. Sex and gender differences in mental disorders. Lancet Psychiatry 2017;4:8-9.

18 Zhong B-L, Liu T-B, Huang J-X, et al. Acculturative stress of Chinese rural-to-urban migrant workers: a qualitative study. PLoS One 2016;11:e0157530.

19 He X, Wong DFK. A comparison of female migrant workers' mental health in four cities in China. Int $J$ Soc Psychiatry 2013;59:114-22.

20 You L, Jin D, Yang H, et al. Psychological health and relevant factors among enterprise employees in Shenzhen. China Journal of Health Psychology 2013;21:1495-7.

21 Zheng L, Tan Q, Xue X. Investigation on mental health and the influencing factors of 1716 migrant workers in Shenzhen City. Strait J Prevent Med 2012;18:9-11.

22 Sun Z. Women's depressed condition and its influencing factors in a factory in Shenzhen. Chinese Primary Health Care 2011;25:32-3.

23 Lin D, Fang X, Lin X, et al. The relationship between mobility, depression and smoking, alcohol use among rural-to-urban femalel migrants in Beijing. Chinese J Clin Psychol 2006;14:614-6.

24 Wang Q, Fu X, Wei Y, et al. The study on anxiety and its influence factors of floating population in Chengdu. J Prevent Med Inform 2007;23:544-6.

25 Shenzhen Statistical Bureau. Bulletin on statistics of economic and social development of Shenzhen. Shenzhen: Statistical Bureau of Shenzhen, 2019.

26 Guo H, Luo Y, Wen J, et al. Suicide ideation among migrant workers in Shenzhen. Chinese Med Modern Distance Educat China 2020;1:149.

27 Kroenke K, Spitzer RL, Williams JB. The PHQ-9: validity of a brief depression severity measure. J Gen Intern Med 2001;16:606-13.

28 Sun XY, Li YX, Yu CQ, et al. [Reliability and validity of depression scales of Chinese version: a systematic review]. Zhonghua Liu Xing Bing Xue Za Zhi 2017;38:110-6.
29 Spitzer RL, Kroenke K, Williams JBW, et al. A brief measure for assessing generalized anxiety disorder: the GAD-7. Arch Intern Med 2006;166:1092-7.

$30 \mathrm{He} \mathrm{X}$, Li C, Qian J, et al. Reliability and validity of a gneralized anxiety scale in general hospital outpatients. Shanghai Archieves of Psychiatry 2010;22:200-3.

31 Hays RD, DiMatteo MR. A short-form measure of loneliness. J Pers Assess 1987;51:69-81.

$32 \mathrm{Li}$. The evaluation and application of the Chinese version of the short-form UCLA loneliness scale (ULS-8). Central South University, 2012.

$33 \mathrm{Li}$ X, Phillips MR, Xu D, et al. Reliability and validity of an adapted Chinese version of Barratt Impulsiveness scale. Chinese Mental Health J 2011;25:610-5.

34 Xiao S. Social support rating scale. Chinese Mental Health J 1993:42-6.

35 Liu J, Li F, Lian Y. Investigation of reliability and validity of the social support scale. J Xinjiang Med Univerity 2008;31:1-3.

36 Su L, Wei B, Ling X, et al. Study on the reliability, validity and norm of scial support scale in Chuang peasants. Modern Prevent Med 2009;36:4411-3.

37 Xie R-H, He G, Koszycki D, et al. Prenatal social support, postnatal social support, and postpartum depression. Ann Epidemiol 2009;19:637-43.

38 Xie Y. Reliability and validity of the simplified coping style questionnaire. Chinese J Clin Psychol 1998;6:114-5.

39 Wang M, Dai X. Chinese meaning in life questionnaire revised in college students and its reliabiity and validity test. Chinese $J$ Clin Psychol 2008;16:459-61.

40 Steger MF, Frazier P, Oishi S, et al. The meaning in life questionnaire: assessing the presence of and search for meaning in life. J Couns Psychol 2006;53:80-93.

$41 \mathrm{R}$ Core Team. R: A language and environment for statistical computing [program], 2013.

42 Revelle WR. psych: procedures for personality and psychological research [program], 2017.

43 Fox J, Weisberg S, Adler D, et al. Package 'car'. Vienna: R Foundation for Statistical Computing, 2012.

44 Bates D, Maechler M, Bolker B, et al. Package 'Ime4'. Convergence2015;12.

45 Ripley B, Venables B, Bates DM, et al. Package 'mass'. Cran $R 2013 ; 538$.

46 Guo Y, Zhao L. The impact of Chinese Hukou reforms on migrant students' cognitive and non-cognitive outcomes. Child Youth Serv Rev 2019;101:341-51.

47 Wang W. Psychological health status in seven provinces in China and brief intervention for alcohol abuse. Shanghai Jiao Tong University, 2014.

$48 \mathrm{Hu}$ J-Z, Hu C-Y, Duan W-D, et al. [Survey on mental disorders among registered residents and non-registered residents in Shenzhen]. Zhonghua Liu Xing Bing Xue Za Zhi 2009;30:543-8.

49 Yan X, Wang X, Qin Y, et al. Prenatal anxiety and its influential factors among floating women from rural area. Chinese $J$ Public Health 2015;31:235-7.

50 Zhou Z, Xu Y, Jin D, et al. Influencing factors of suicide ideation among migrant service workers in Shenzhen City. Chinese J Public Health 2016;32:948-52.

51 Liu X, Liu Y, Pan R, et al. Anxiety and depression survey analysis among migrant workers of leather industry in Shenzhen. $J$ Med Theory Prac 2012;25:1972-4.

52 Hu W, Wang R. Which Chinese cities are more inclusive and why? Cities 2019;86:51-61.

53 Zhong B-L, Liu T-B, Chiu HFK, et al. Prevalence of psychological symptoms in contemporary Chinese rural-to-urban migrant workers: an exploratory meta-analysis of observational studies using the SCL90-R. Soc Psychiatry Psychiatr Epidemiol 2013;48:1569-81.

54 Lin Y, Zhang Q, Chen W, et al. The social income inequality, social integration and health status of internal migrants in China. Int $J$ Equity Health 2017;16:139.

55 Magnani E, Zhu R. Gender wage differentials among rural-urban migrants in China. Reg Sci Urban Econ 2012;42:779-93.

56 Xu S, Tian L. An empirical study on gender differences in income of migrant workers from Jiangsu, Zhejiang and Shanghai. Guizhou Social Sciences 2015;305:34-9.

57 Danke L, Tasang MC. Household decisions and gender inequality in education in rural China. China: An Inter J 2003;1:224-48.

58 Wei J, Luo Z, Weng Z. Impact of human capital factors on employment and income levels of nascent migrant workers. Economic Research Guide 2013;191:151-3. 